\title{
A modified murine model of systemic sclerosis: bleomycin given by pump infusion induced skin and pulmonary inflammation and fibrosis
}

\author{
Minrui Liang 1,2,7, Jiaoyan Lv ${ }^{3,7}$, Linlin Zou ${ }^{3}$, Wei Yang ${ }^{3}$, Yingluo Xiong ${ }^{3}$, Xiangjun Chen ${ }^{2,4}$, Ming Guan ${ }^{2,5}$, Rui He $\mathrm{He}^{3,6}$ \\ and Hejian Zou ${ }^{1,2}$
}

Daily subcutaneous (sc) injection of bleomycin (BLM) causes dermal fibrosis but rarely causes lung changes in mice. There are also significant disadvantages to this traditional model for systemic sclerosis, including a variable distribution of lesions and a requirement for repetitive procedures. The present study was undertaken to develop a convenient method of BLM administration that yields stable dermal inflammation and fibrosis with extensive and reproducible interstitial lung disease (ILD) in mice. Osmotic minipumps containing BLM $(150 \mathrm{mg} / \mathrm{kg})$ or saline were implanted sc in C57BL/6 mice and the drug was delivered as a continuous infusion over $1 \sim 4$ weeks. The time course of morphological features, collagen content, and pro-inflammatory cytokine expression in the skin and the lungs were analyzed. Pathological examination demonstrated dominant inflammatory infiltrates at week 1 and significant fibrosis at week 4. Decreased microvessel density and increased myofibroblast counts were observed in the skin of BLM-treated mice at week 4. In addition, there were obvious increases in dermal infiltration of $\mathrm{CD} 45^{+}$leukocytes, including $\mathrm{F} 4 / 80^{+}$macrophages, $\mathrm{Gr}-1^{+}$neutrophils, and $\mathrm{CD}^{+}{ }^{+} \mathrm{T}$ lymphocytes in BLM-treated mice. IL-1 $\beta$, IL-4, and CXCL2 transcripts were continually upregulated by BLM in the skin and lung tissues. In addition, lungs from BLM-treated mice showed significant inflammatory infiltrates and confluent subpleural fibrosis at week 4 . In conclusion, this modified murine model for drug-induced systemic inflammation and fibrosis uses a single procedure and provides reproducible skin and lung lesions, mimicking human systemic sclerosis (SSc) with ILD-like manifestation.

Laboratory Investigation (2015) 95, 342-350; doi:10.1038/labinvest.2014.145; published online 15 December 2014

Systemic sclerosis (SSc) is a multisystem autoimmune disease characterized by three features: inflammation and autoimmunity, vasculopathy, and fibrosis of skin and internal organs. Interstitial lung disease (ILD) is one of the significant complications of SSc with high mobility and mortality. Despite growing effort in the field of SSc, the pathogenesis of SSc is far from clear.

Both innate and adaptive immunities are documented to contribute to the initiation and the development of SSc. Examination of the skin lesions in SSc patients showed that immunological alterations such as T-cell activation, altered CD4/CD8 ratio, and the recruitment of macrophages. ${ }^{1}$ The immune cells contribute to the release of several cytokines such as interleukin-1 (IL-1) $\beta$, IL-4, IL-6, IL-13, and TGF- $\beta$, which exert their pro-inflammatory and pro-fibrotic effects on the endothelium and fibroblasts. The role of Th17 cell in the pathogenesis of SSc is debatable. ${ }^{2-6}$ Interestingly, integrin-modulating therapy was shown to prevent not only fibrosis but also autoimmunity in stiff skin syndrome (SSS) mouse models, indicating that alterations in cell-matrix interactions could initiate and sustain inflammatory and profibrotic programming. ${ }^{7}$ More importantly, patients with inflammatory skin transcript signature are more likely to respond to immunosuppressive therapy and demonstrated clinical improvement. ${ }^{8}$ However, no effective treatment has yet been approved to cure SSc. The study of SSc is hindered

\footnotetext{
'Division of Rheumatology, Huashan Hospital, Fudan University, Shanghai, China; ${ }^{2}$ Institute of Rheumatology, Immunology and Allergy, Fudan University, Shanghai, China; ${ }^{3}$ Department of Immunology, School of Basic Medical Sciences, Fudan University, Shanghai, China; ${ }^{4}$ Division of Neurology, Huashan Hospital, Fudan University, Shanghai, China; ${ }^{5}$ Department of Clinical Laboratory, Huashan Hospital, Fudan University, Shanghai, China and ${ }^{6}$ Biotherapy Research Center, Fudan University, Shanghai, China

Correspondence: Professor R He, PhD, Department of Immunology, School of Basic Medical Sciences, Fudan University, 138 no. Yixueyuan Road, Shanghai 200032, China or Dr H Zou, MD, PhD, Division of Rheumatology, Huashan Hospital, Fudan University, 12 no. Wulumuqi Zhong Road, Shanghai 200040, China. E-mail: ruihe@fudan.edu.cn or hjzou@fudan.edu.cn

${ }^{7}$ These authors contributed equally to this work.

Received 1 September 2014; revised 13 October 2014; accepted 22 October 2014
} 
by a lack of animal models that recapitulate the etiology of this complex disease.

Therefore, an animal model is in urgent need to facilitate the understanding of the pathogenic mechanism in order to develop novel therapeutic targets. The bleomycin (BLM)induced SSc mouse model is the most widely used, which encompasses all three features of the human disease. ${ }^{9}$ Traditionally, daily subcutaneous (sc) injection of BLM for $4 \sim 6$ weeks is used to induce local skin inflammation and fibrosis. However, this traditional model is associated with significant disadvantages including a variable distribution of lesions, rare involvement of the lung, and a requirement for repetitive procedures. While bolus injection leads to the fluctuation of plasma drug level, which depends on the halflife of the agent relative to the length of intervals between injections and may explain the intermittent efficacy, high peak in concentration may trigger unwanted side effects, such as weight loss and high mortality. We propose that controlled continuous drug exposure can obviate these problems.

Our study aimed to develop a stable and convenient SSc murine model that more closely mimics human SSc. We therefore implanted osmotic minipumps containing BLM sc in C57BL/6 mice and developed a SSc murine model involving both the skin and the lung. We herein describe the time course of morphological changes in the skin and the lung following the treatment with osmotic minipumps with BLM in detail. Furthermore, we present a detailed analysis of local inflammatory infiltrates and pro-inflammatory cytokine expression.

\section{MATERIALS AND METHODS Animal Study}

Wild-type (WT) C57BL/6 mice aged $6 \sim 8$ weeks were purchased from the Chinese Academy of Science (Shanghai, China) and kept in a pathogen-free environment. Experimental procedures were in accordance with the Animal Care and Use committee at Fudan University, Shanghai, China. Osmotic minipump (Alzet 2004, DURECT, Cupertino, CA, USA) contains either BLM (Nippon Kayaku, Tokyo, Japan) at the concentration of $150 \mu \mathrm{g} / \mathrm{mg}$ dissolved in $247 \mu \mathrm{l}$ saline or the saline vehicle of the same volume, and delivers the content at $0.25 \pm 0.05 \mu \mathrm{l} / \mathrm{h}$ for $1 \sim 4$ weeks. On day 0 , mice were anesthetized with $0.75 \%$ pentobarbital sodium, and the osmotic minipumps with either BLM or saline were implanted sc at the right back after shaved. Mice were killed, and then systemically perfused via the left ventricle with PBS at weeks 1, 2, 3, or 4, respectively. The serum, skin, and lung tissues were collected for further analysis. Daily injection of BLM sc to the WT C57BL/6 mice aged $6 \sim 8$ weeks for 4 weeks to induce the traditional SSc mouse model as control was performed as previous described. ${ }^{9}$

\section{Histopathology}

The skin at the lesion sites and two upper lobes of the right lung tissue were harvested from the killed mice. Specimens were fixed in $10 \%$ neutral buffered formalin solution and embedded in paraffin. Multiple $4-\mu \mathrm{m}$ sections were stained with hematoxylin and eosin (H\&E) for histopathological analysis or with Masson's trichrome for the evaluation of collagen content and distribution.

Dermal thickness was measured after taking photographs under a light microscope (Leica, Germany; DMI4000B) for the H\&E sections. The dermal thickness was defined as the mean distance between the epidermal-dermal junction and the dermal-subcutaneous fat junction measured at 5 different skin sections of each mouse with Image J. ${ }^{10}$ This analysis was performed by two independent observers.

The Ashcroft scale for the evaluation of lung fibrosis is the analysis of stained histological samples by visual assessment. ${ }^{11}$ The grading criteria were as follows: (A) Grade 0, normal lung. (B) Grade 1, isolated alveolar septa with gentle fibrotic changes. (C) Grade 2, fibrotic changes of alveolar septa with knot-like formation. (D) Grade 3, contiguous fibrotic walls of alveolar septa. (E) Grade 4, single fibrotic masses. (F) Grade 5, confluent fibrotic masses. (G) Grade 6, large contiguous fibrotic masses. (H) Grade 7, air bubbles. (I) Grade 8, fibrous obliteration. This analysis was also performed by two independent observers.

For immunohistochemical analysis, the sections were treated with $3 \%$ hydrogen peroxide for $10 \mathrm{~min}$ at room temperature to block endogenous peroxidase. Subsequently, the sections were incubated with the antibody against CD45, $\mathrm{F} 4 / 80, \mathrm{Gr}-1, \mathrm{CD} 3, \alpha-\mathrm{SMA}$, or CD31 overnight at $37^{\circ} \mathrm{C}$, and then incubated at room temperature for $1 \mathrm{~h}$ with horseradish peroxidase-conjugated goat anti-rat/rabbit IgG (Dako, Glostrup, Denmark) secondary antibody. Sections were washed three times with PBST buffer between each incubation. After development with $3,3^{\prime}$-diaminobenzidine tetrahydrochloride and hydrogen peroxide, the sections were counterstained with hematoxylin. These primary monoclonal antibodies (mAbs) mentioned above were all obtained from Abcam (Cambridge, MA, USA).

\section{Sircol Collagen Assay}

The skin tissues and the left lopes of lung tissues were homogenized in $1 \mathrm{ml}$ pepsin $(0.1 \mathrm{mg} / \mathrm{ml}$ in $0.5 \mathrm{M}$ acetic acid) and digested by shaking at room temperature overnight. After that, the supernatant was collected by centrifuging at 12000 r.p.m. for $20 \mathrm{~min}$ at room temperature. The total collagen content of the skin and the lung was measured by the Sircol collagen dye-binding assay, according to the manufacturer's instructions (Biocolor Ltd., Carrickfergus, $\mathrm{UK})$.

\section{Quantitative RT-PCR}

Total RNA was extracted from the skin and lung tissue samples in $1 \mathrm{ml}$ TRIZOL regent (Invitrogen, Carlsbad, CA, USA) according to the manufacturer's instructions. RNA was reverse-transcribed using the PrimeScript RT reagent kit (TaKaRa Bio, Otsu shiga, Japan). Real-time RT-PCR was 
performed on an ABI 7500 Fast Real-Time PCR System (ABI, Foster City, CA, USA) using SYBR Green PCR Master mix (TaKaRa). All data were normalized to the expression of housekeeping gene $\beta$-actin.

\section{ELISA}

The concentration of TGF- $\beta 1$ in the serum was determined by ELISA following the manufacturer's guidelines (eBioscience, SanDiego, CA, USA).

\section{Statistical Analysis}

Statistical significance was analyzed using the Student's $t$-test. A $P$-value less than 0.05 was considered statistically significant.

\section{RESULTS}

\section{Delivery of BLM by Skin-Embedded Osmotic Minipump Induces Dermal Inflammation and Fibrosis}

To evaluate the potentiality of BLM delivered by osmotic minipump to induce SSc-like manifestations in the mouse, C57BL/6 mice were sc implanted an osmotic minipump with BLM $(150 \mathrm{mg} / \mathrm{kg}$ total dose dissolved in $247 \mu \mathrm{l}$ saline, $0.25 \pm 0.05 \mu \mathrm{l} / \mathrm{h}$ ), to the shaved skin on the right back. Saline in the same volume delivered by the osmotic minipump was used for the control group. No mortality and weight loss was noted in both experimental and control groups (data not shown). As shown in the protocol, mice were killed at different time points, the skin and the lung samples were then collected for further examination (Figure 1). Routine H\&E, Masson's trichrome staining, and immunohistochemical analysis were performed for the skin lesion to explore the dynamic changes in skin morphology following the implantation of osmotic minipumps with BLM.

Pathological examination revealed significant swollenness, thickening, and inflammatory infiltrates in the skin 1 week after implantation of minipumps with BLM (Figure 2a).

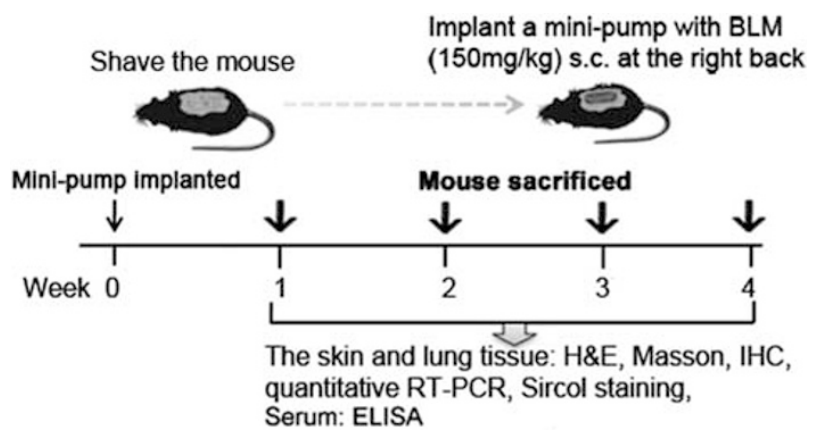

Figure 1 Protocol for establishing systemic sclerosis (SSc) murine model. Bleomycin (BLM) was administered as a continuous subcutaneous (sc) infusion from an osmotic minipump $(150 \mathrm{mg} / \mathrm{kg}$, dissolved in $247 \mu \mathrm{l}$ saline). Implantation of osmotic minipumps filled with BLM or saline were conducted and the mice were killed for further analysis at weeks 1, 2, 3, and 4 , respectively.
Abundant infiltrating inflammatory cells were observed in the skin lesions, especially in the dermal and subcutaneous layer, whereas no inflammatory infiltrate was observed in the skin of the control group. There were gradual decreases in skin swollenness and inflammatory infiltrates since week 2 ; in contrast, skin fibrosis was observed in week 3 and became severer at week 4 , which was histologically characterized by thinner epidermis, deposition of abundant homogenous collagen bundles, and accumulation of fibroblasts in the dermis. Moreover, we observed that the adipose and glandular tissues were reduced and replaced by collagen bundles (Figure 2a). This phenomenon closely mimicked the typical progression phases in the skin of SSc including (1) edematous phase and (2) fibrosis phase. During the whole observation time, there was no inflammation or fibrosis observed in the skin of the control group, suggesting that the embedded pump itself was not able to cause the features of SSc skin lesion. Accordingly, the skin lesion from BLM-treated mice exhibited significantly increased skin thickness as compared with those from control mice $(852.5 \pm 85.7 v s 270.3 \pm 7.7 \mu \mathrm{m}$ at 1 week, $P<0.05 ; 852.3 \pm 36.1 v s 278.8 \pm 26.3 \mu \mathrm{m}$ at 2 week, $P<0.05 ; 864.2 \pm 30.1$ vs $307.4 \pm 38.5 \mu \mathrm{m}$ at 3 week, $P<0.05$; $884.3 \pm 17.3$ vs $273.8 \pm 21.7 \mu \mathrm{m}$ at 4 week, $P<0.05$; Figure 2b). The significant increase in the content of collagen in the skin lesions from BLM-treated mice was only detected in week 4 (Figure 2c).

As reduced dermal microvessels is a hallmark of the vasculopathy in human SSc, we stained the skin sections with a pan-endothelial marker CD31 to analyze the density of microvessels. A significant decrease in the number of CD31positive dermal capillaries was found in mice receiving the treatment of osmotic minipump with BLM at week 4 $(P<0.05$; Figure $3 \mathrm{a}$ and $\mathrm{b})$. In addition, the treatment of BLM for 4 weeks caused a significant increase in the number of $\alpha$-SMA-positive dermal myofibroblasts as shown in Figure 3a and $\mathrm{c}(P<0.05)$. These data collectively showed that BLM delivered by osmotic minipumps could induce three typical SSc dermal manifestations in mice: inflammation, fibrosis, and vasculopathy.

\section{Delivery of BLM by Skin-Embedded Osmotic Minipumps Leads to Inflammatory Infiltrates in the Skin}

To further characterize the inflammatory infiltrates in the skin, we performed immunohistochemical analysis of the skin biopsies. Abundant $\mathrm{CD} 45^{+}$leucocytes were shown to accumulate in the skin lesions of experimental group at week 1 , especially in the dermal and sc layer, and partial resolution of $\mathrm{CD}_{4} 5^{+}$infiltration was noted at week 4 , which was consistent with the H\&E staining for the skin sections (Figure 4a). Further identification of the types of infiltrating cells using a panel of mAbs showed a strikingly increased cells expressing macrophage marker F4/80 in the skin lesions of BLM-treated group, predominantly in the deep dermal layer, which peaked at week 1 . Very few $\mathrm{F} 4 / 80^{+}$macrophages were observed in the skin of the control group (Figure 4b). The 
a

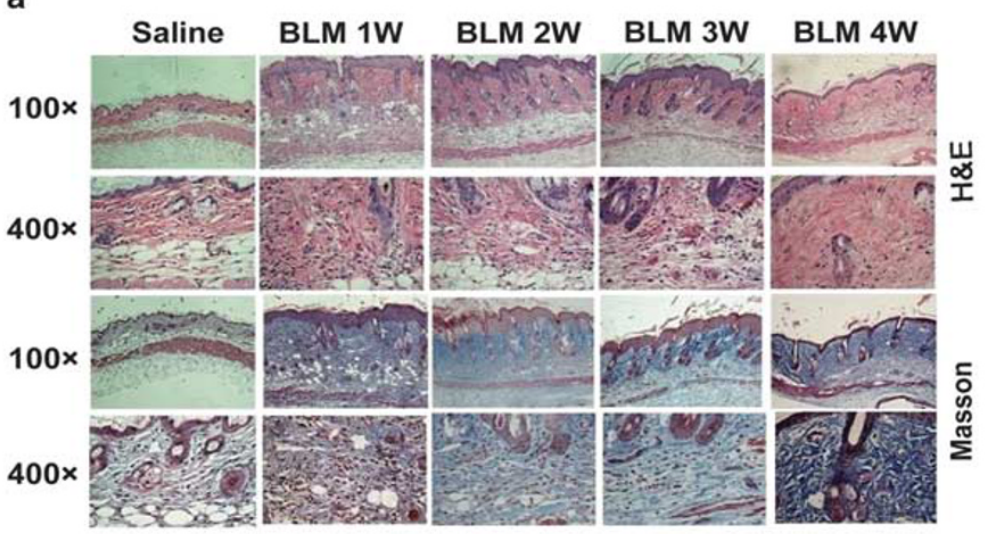

b

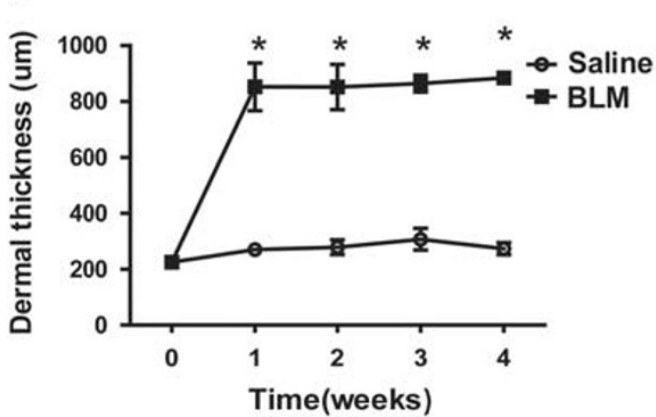

C

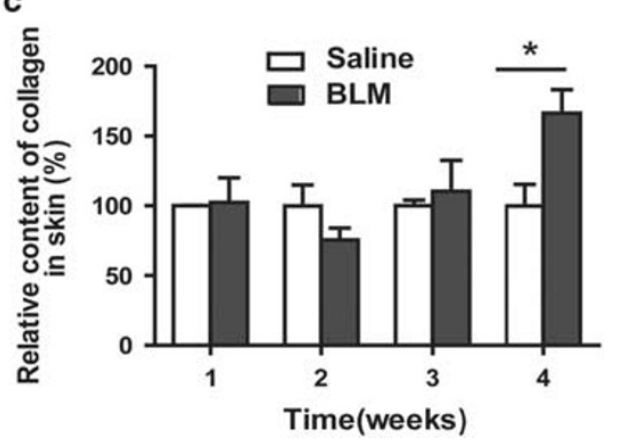

Figure 2 Time course of changes in skin morphology and collagen content following the implantation of osmotic minipumps with bleomycin (BLM) subcutaneous (sc) in C57BL/6 mice. (a) C57BL/6 mice receiving either BLM or saline by osmotic minipumps, and killed at weeks 1, 2, 3, and 4. Skin tissues at the lesion sites were collected for hematoxylin and eosin (H\&E) and Masson's trichrome staining $(\times 100$ and $\times 400)$. (b) Dermal thickness was measured with Image J by two independent individuals. Dermal thickness was shown to be significantly increased since week 1 and peaked at week 4 , whereas mice treated with saline (continuous infusion) did not show an increase in dermal thickness. (c) Skin collagen content was measured with Sircol collagen assay. Mice receiving continuous BLM infusion by minipumps exhibited increased collagen content in the skin compared with salinetreated control at week 4 . Values are the means of $4 \sim 8$ mice \pm s.e. from three independent experiments. ${ }^{*} P<0.05$.
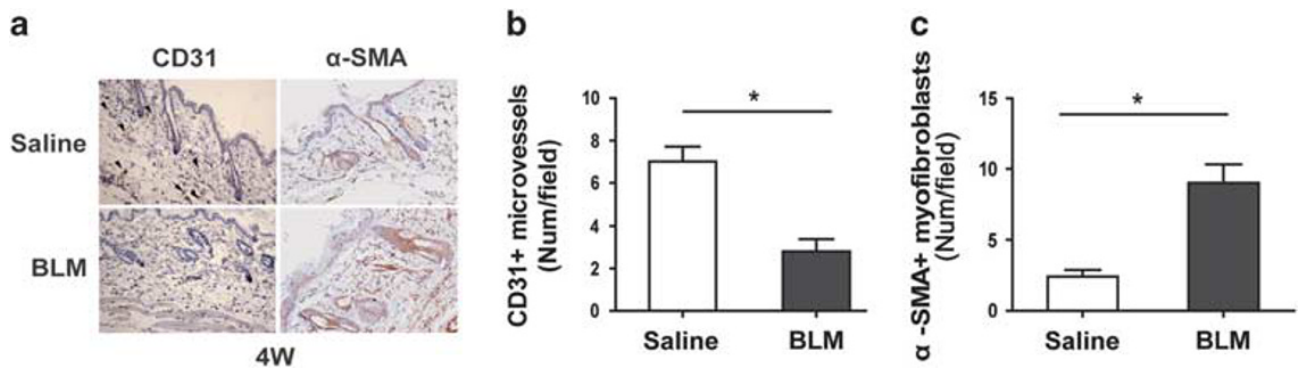

Figure 3 Decreased microvessel density and increased myofibroblast counts in the skin of mice following the implantation of minipumps with bleomycin (BLM). (a) Representative microphotographs of skin sections from the mice following the implantation of minipumps with BLM ( $n=4 \sim 8)$ or saline ( $n=4 \sim 8)$ immunostained for the pan-endothelial cell marker CD31 or $\alpha$-smooth muscle actin ( $\alpha$-SMA) are shown. CD31-positive microvessels are indicated by arrows. Myofibroblasts were identified as $\alpha$-SMA-positive spindle-shaped cells. Original magnification $\times 200$. (b) Data are mean \pm s.d. of CD31-positive microvessel counts per high-power field. ${ }^{*} P<0.05$. (c) Quantification of myofibroblasts in the dermis of mice treated with saline or BLM. Data are mean \pm s.d. of $\alpha$-SMA-positive myofibroblast counts per high-power field. ${ }^{*} P<0.05$. These data were repeated in three independent experiments.

presence of neutrophil marker Gr-1 was observed in the dermis of experimental group; however, no Gr-1-positive cells were observed in the skin of control group (Figure 4c). Positive CD3 staining was observed in the both epidermis and dermis of the experimental group; however, fewer numbers of immunoreactive cells for $\mathrm{CD} 3$ were seen in the control group (Figure 4d). These data indicated the possible involvement of leukocytes including $\mathrm{F} 4 / 80^{+}$macrophage, Gr- $1^{+}$neutrophil, and $\mathrm{CD} 3^{+} \mathrm{T}$ cell in the BLM-induced SSc murine model. 
a

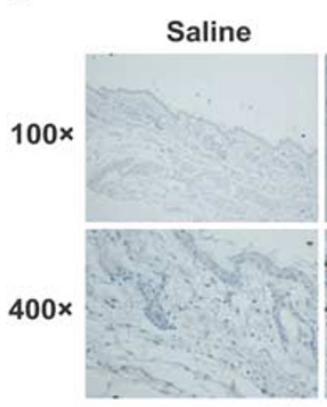

c

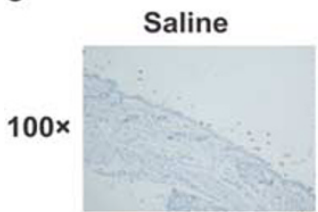

$400 x$

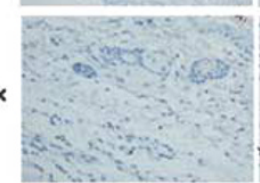

BLM 1W

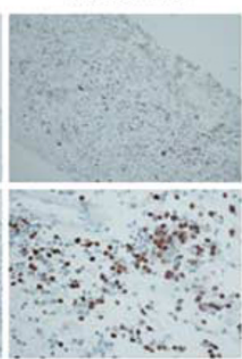

BLM 1W
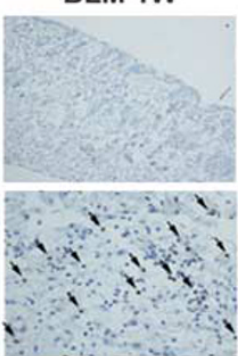

BLM 4W

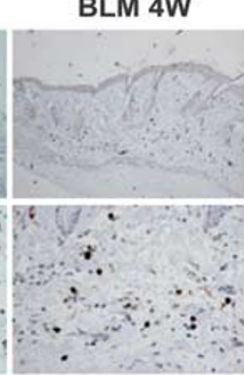

BLM 4W

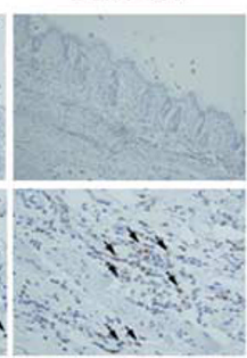

b

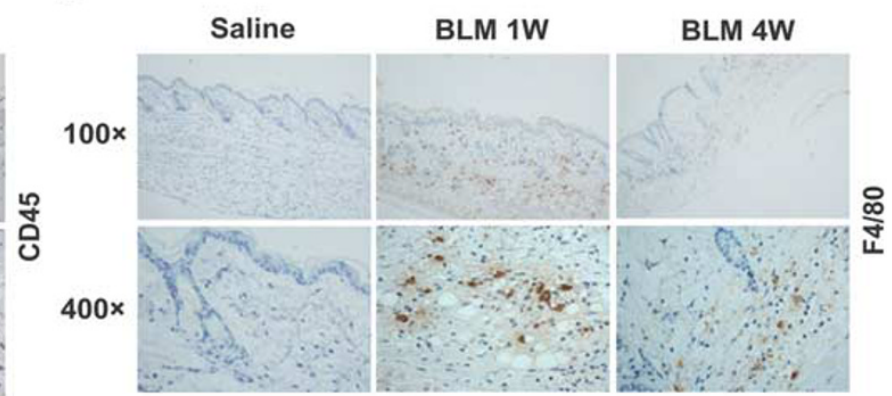

d

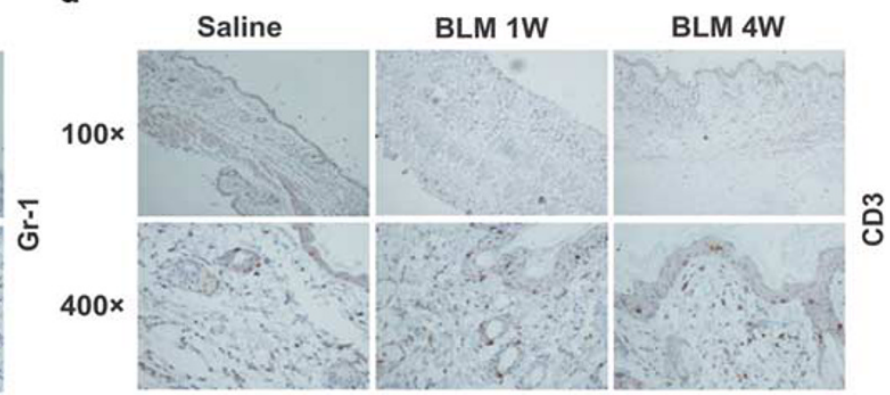

Figure 4 Cellular infiltrates in the skin lesions following the implantation of osmotic minipumps with BLM sc in C57BL/6 mice. Bleomycin (BLM) or saline was administered as a continuous subcutaneous (sc) infusion from an osmotic minipump, and then the mice were killed at the indicated time points and the skin tissues were collected for immunohistochemical analysis. Positive cells staining with leukocyte marker CD45 (a), macrophage marker F4/80 (b), neutrophil marker Gr-1 (c), and T lymphocyte marker CD3 (d) were seen in the skin lesions after BLM treatment, whereas fewer positive cells could be detected in the skin of saline control.

Delivery of BLM by Skin-Embedded Osmotic Minipumps Upregulates the Pro-inflammatory Cytokines in the Skin

Emerging evidence suggests that a panel of cytokines originated from leukocytes and tissue cells contribute to the inflammation and subsequent fibrosis in SSc. To determine whether implantation of osmotic minipumps with BLM could lead to the local inflammation in the skin, we examined the levels of pro-inflammatory cytokines in the skin lesions. According to the morphological changes in the skin, we observed above significant inflammation and fibrosis at weeks 1 and 4, respectively; therefore, we collected the skin biopsies for further cytokine transcript analysis at weeks 1 and 4.

As shown in Figure $5 \mathrm{a}$, the expression levels of IL-1 $\beta$, IL-4, IL-6, and CXCL2 mRNA were significantly increased in the skin from BLM-treated mice compared with those from control mice at week 1 . In contrast, there was no significant increase in the gene expressions of IL-13, IL-17A, IL-22, or CCL17 (Figure 5a). The transcript levels of IL-1 $\beta$, IL-4, IL-13, IL-22, CXCL2, and CCL17, but not of IL-6 or IL-17A, were increased in the affected sites of the skin 4 weeks after implantation of minipumps with BLM (Figure 5b). It is well documented that IL-4 is a critical polarizing cytokine for Th2 cell differentiation, and Th2 cell exerts its effect on fibrosis mainly by IL-13. ${ }^{12}$ There were no changes in the mRNA level of IFN- $\gamma$ at weeks 1 and 4 (data not shown). The transcript level of IL-17A in the skin of experimental group was relatively higher than that in control group at week 4, but not reaching statistical significance. Our data therefore supported a predominant Th2 response in this SSc model. In addition, the expressions of chemokines CXCL2 and CCL17 were shown to be induced in the skin lesion following implantation of minipumps with BLM (Figure 5b), which strongly supported the cellular infiltration of neutrophils and $\mathrm{T}$ cells in the skin lesion according to their preferential chemotactic ability. ${ }^{13-16}$

\section{Delivery of BLM by Skin-Embedded Osmotic Minipumps Induces Interstitial Lung Disease-Like Manifestation}

SSc is a systemic autoimmune disease with multiple organs involved, and ILD is one of the most significant complications in SSc. TGF- $\beta 1$ is widely considered to have a central role in fibrosis. As shown in Figure $6 \mathrm{a}$, implantation of minipumps with BLM led to high level of TGF- $\beta 1$ in serum at week $4(P<0.05)$, suggestive of a systemic response elicited by BLM through skin-embedded osmotic minipumps.

To evaluate the dynamic changes in morphology in the lungs of C57BL/6 mice receiving BLM delivered by osmotic minipumps, right lungs were removed for pathological analysis at indicated time. Compared with the control group, the inflammatory infiltrates and epithelial atypia were shown to be pronounced in mice administrated with BLM around 
a
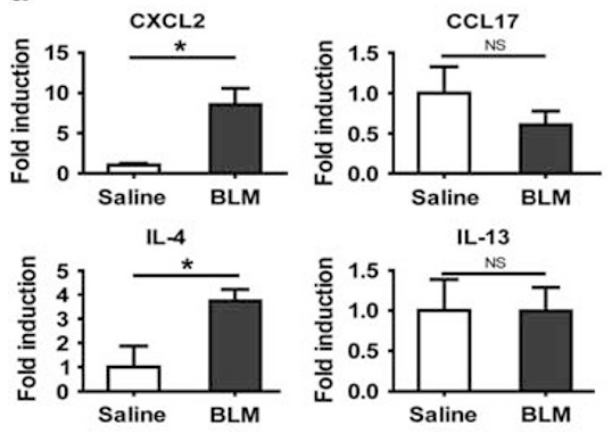

b
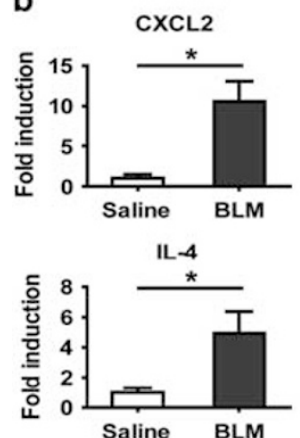
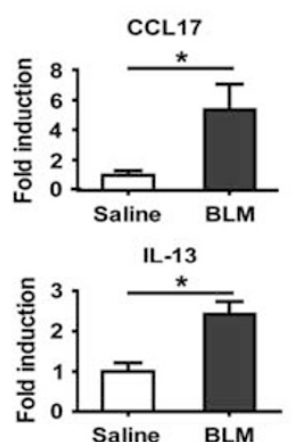
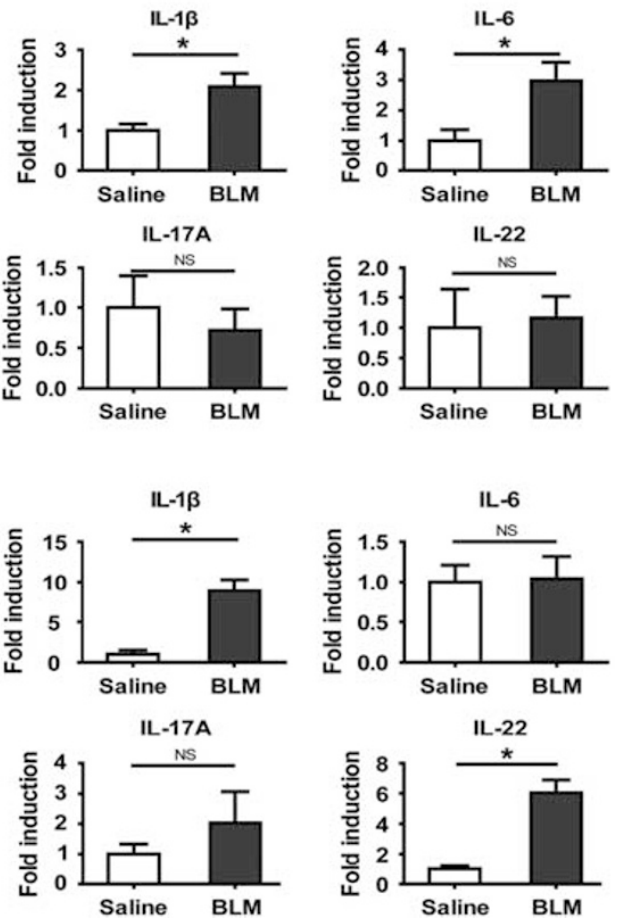

Figure 5 The expressions of pro-inflammatory cytokines in the skin lesion following the implantation of osmotic minipumps with bleomycin (BLM) subcutaneous (sc) in C57BL/6 mice. Quantitative RT-PCR analysis of CXCL2, CCL17, IL-1 $\beta$, IL-6, IL-4, IL-13, IL-17, and IL-22 levels in the skin biopsies from C57BL/6 mice treated with BLM or saline through osmotic minipumps at week 1 (a) and week 4 (b). Pooled results of experiments using BLM-treated mice and saline controls $(n=4 \sim 8)$ from three independent experiments in triplicate. Bars represent mean \pm s.e. NS, not significant; ${ }^{*} P<0.05$.

week 3. And at week 4, there was significant inflammatory infiltrate and extensive consolidation of lung parenchyma with the loss of alveolar architecture and increased cellularity, and these lesions mainly appeared subpleurally (Figure 6b). In order to compare with the traditional SSc model, C57BL/6 mouse was injected with BLM sc daily for consecutive 4 weeks as control at the same time. ${ }^{9}$ In contrast with BLMpump-induced SSc mouse, little inflammation or fibrosis was observed in the lung tissues of SSc mouse treated with daily sc injection of BLM (Figure 6b). The increased CD45-positive leukocytes and the loss of integrity of CD31-positive pulmonary capillaries were also found in the lungs of the mouse receiving the treatment of osmotic minipump with BLM at week 4 (Figure 6c), which supported the involvement of pro-inflammatory infiltration and vasculopathy in the progression of the lung disease induced by systemic BLM. To compare the extent of fibrosis at subpleural sites, H\&Estained sections were further analyzed for fibrosis scoring by Ashcroft's method. ${ }^{11}$ Implantation of minipumps with BLM led to a time-dependent increase in Ashcroft's fibrosis score, and became much severer at week $4(P<0.05)$ compared with that of saline control (Figure 6d). In addition, the Ashcroft score of the lung tissues in SSc mouse induced by daily injection of BLM was comparable with that in saline control mouse (Figure 6d). These data indicated that the mice treated with BLM through an osmotic minipump could resemble human SSc with ILD-like manifestation.

To further evaluate whether osmotic minipump implantation with BLM can prime mice to develop pulmonary inflammation, we examined the transcript levels of proinflammatory cytokines in the lung tissues. Figure 6e showed that the transcript levels of CXCL2, CCL17, IL-1 $\beta$, and IL-4 were significantly increased in the lungs of the mice treated with BLM through osmotic minipump at week 4, although there were no significant changes in the expressions of CXCL2, CCL17, IL-1 $\beta$, IL-6, IL-4, IL-13, IL-17A, or IL-22 at week 3 when compared with those of saline control (data not shown), indicating that significant pulmonary inflammation occurred later around week 4 following the implantation of osmotic minipumps with BLM.

\section{DISCUSSION}

This study demonstrates a modified SSc murine model that employs the implantation of osmotic minipumps with BLM in the skin of C57BL/6 mice, which elicits tissue inflammation and fibrosis in both local skin and the distant lung tissue, pathologically mimicking the human SSc with ILDlike manifestation.

Traditionally, daily injection of BLM sc for $4 \sim 6$ weeks is widely used to establish a SSc model; however, this model is 
a

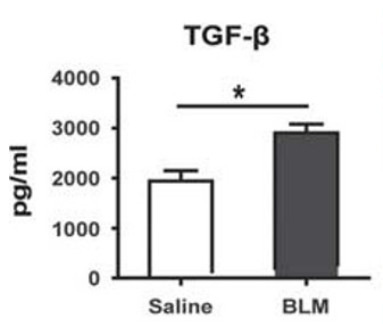

b

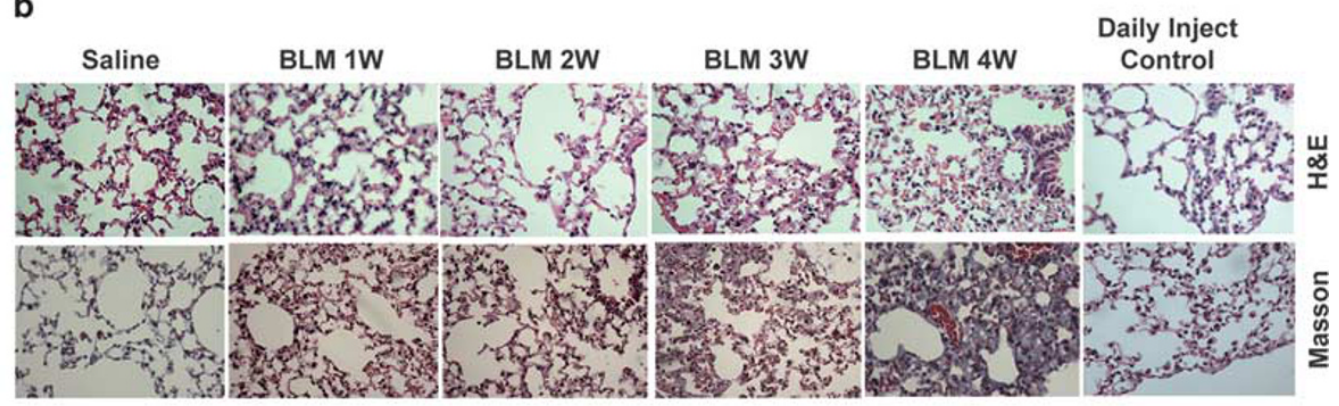

C

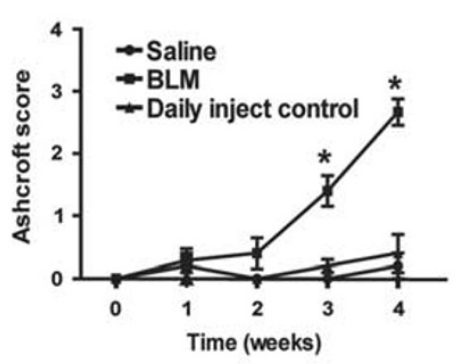

d

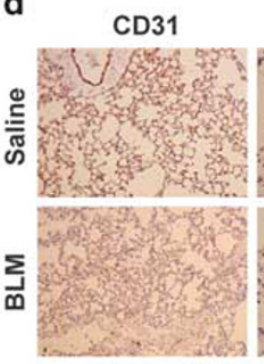

CD45

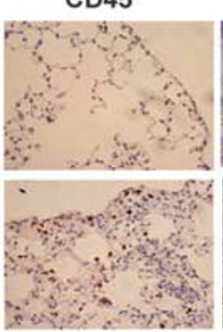

$\mathrm{F} 4 / 80$

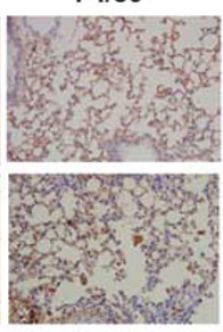

Gr-1

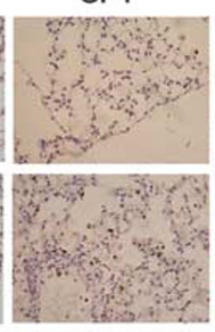

CD3

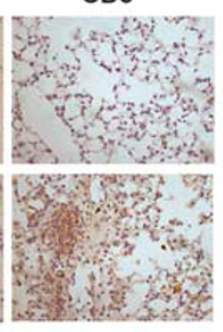

e
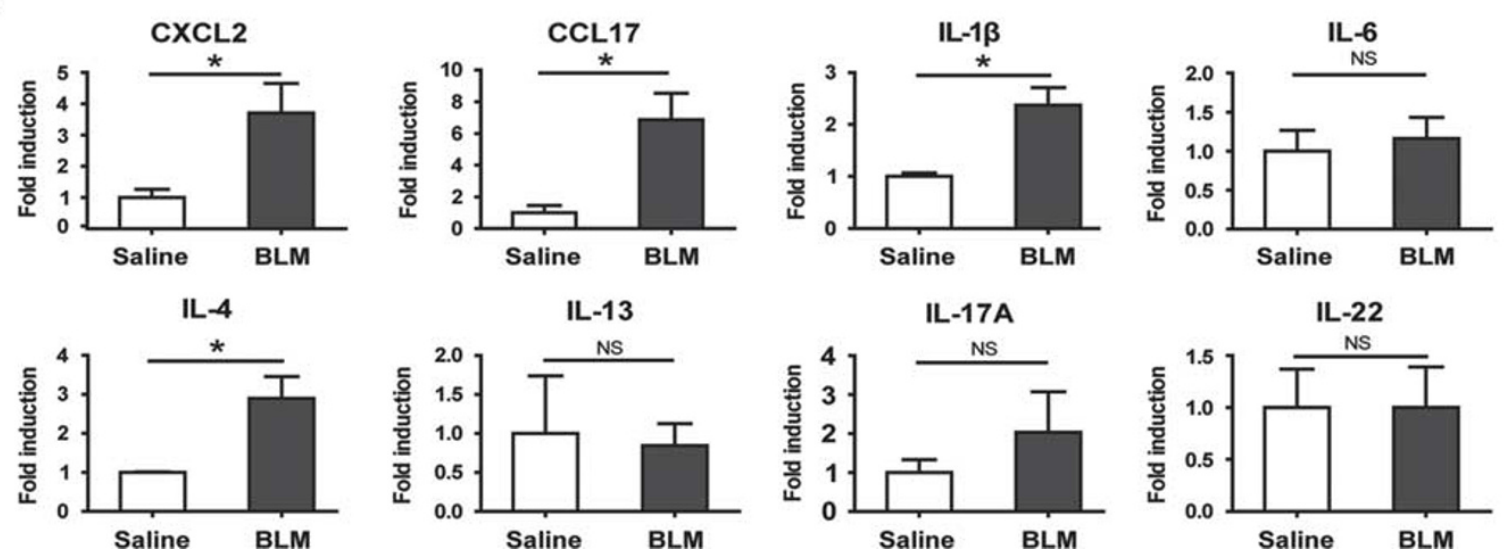

Figure 6 The changes in serum TGF- $\beta$ 1, lung morphology, and pro-inflammatory cytokine expressions in the lung tissues following the implantation of osmotic minipumps with bleomycin (BLM) subcutaneous (sc) in C57BL/6 mice. (a) ELISA assay showed elevated TGF- $\beta$ in the serum from the mice treated with BLM via osmotic minipump at week 4. Data represent the mean \pm s.e. $(n=4 \sim 8)$. ${ }^{*} P<0.05$. (b) C57BL/6 mice receiving either saline or BLM by osmotic minipumps, and the traditional systemic sclerosis ( $\mathrm{SSc}$ ) model was established by daily injection of BLM sc as indicated as 'daily inject control'. The lung tissues were collected for hematoxylin and eosin (H\&E) (the upper two lines) and Masson's trichrome staining (the lower two lines; $\times 100$ and $\times 400$ ). (c) Immunohistochemical analysis was performed on the C57BL/6 mice receiving the treatment of BLM or saline via osmotic minipump for 4 weeks. Positive cells staining with leukocyte marker CD45 and endothelial marker CD31 were as indicated ( $\times 200)$. (d) The Ashcroft score was used for evaluating lung fibrosis on stained histological samples by visual assessment. (e) Quantitative RT-PCR analysis of CXCL2, CCL17, IL-1 $\beta$, IL-6, IL-4, IL-13, IL-17A, and IL-22 levels in the lung tissues from C57BL/6 mice treated with BLM or saline via osmotic minipump at week 4. Data were pooled from three independent experiments $(n=4 \sim 8)$. Data represent mean \pm s.e. NS, not significant; ${ }^{*} P<0.05$.

associated with significant disadvantages including limited lung involved, variable skin lesion, and a requirement for repetitive procedures. Although intravenous or intraperitoneal injection of BLM leads to SSc-like changes especially lung fibrosis in mice, it causes high mortality. Therefore, a modified protocol to establish a SSc mouse model is in urgent need. Herein, the strength of the modified protocol in the present study is underlined that single procedure that implantation of an osmotic minipump with BLM could produce reproducible SSc-ILD-like manifestation in mice and, moreover, there was no weight loss or mortality in this modified murine model. Therefore, this newly addressed protocol is an obvious improvement.

Overall, this murine model demonstrated the significant tissue inflammation and persistent infiltration of immune cells, loss of microvessels and ultimate fibrosis, which closely resembled the three typical characteristics in human SSc. Histological examination of the skin of patients with SSc 
during the early edematous inflammatory phase of the disease demonstrates the presence of mononuclear cell infiltrates containing $\mathrm{T}$ cells preceding the development of fibrosis and overt vasculopathy. ${ }^{17,18}$ Abundant leukocytes including $\mathrm{T}$ cells, macrophages, and neutrophils were observed in the dermal and sc layers of the mouse receiving the BLM treatment via minipump at week 1 , and the inflammatory infiltrates decreased over time; however, significant fibrosis was seen at week 4 . Thus, the time course of morphological analysis demonstrated that this modified SSc murine model resembles the typical phases in the progression of SSc in human including both the edematous phase and the fibrotic phase. Moreover, the inflammatory infiltrates were present particularly in the dermis of skin and in the subpleural areas of lung, which was closely correlated with the subsequent fibrotic areas. In addition, the pattern of impaired expressions of pro-inflammatory cytokines and the accumulation of immune cells in the skins and the lungs further mimicked the changes in SSc patients, which collectively indicated the involvement of inflammation in this mouse model. Thus, the fibrosis in this BLM-pump-induced SSc mouse was likely driven by inflammation.

In agreement with previous reports that a murine model of lung fibrosis induced by implanting an osmotic minipump containing BLM, ${ }^{19,20}$ we confirmed this finding based on our observation of the pathological changes in the lung. Compared with the lung changes induced by intratracheal administration or multiple sc injections of BLM, BLM given by osmotic minipump led to a greater increase in lung hydroxyproline and much extensive pleural surface involvement. ${ }^{19,20}$ As the skin and lung exhibit the low activity of BLM hydrolase, a cytosolic aminopeptidase, ${ }^{21}$ they are more sensitive to BLM than other organs. Therefore, systemic BLM given via osmotic minipump leads to skin and lung infiltration of activated inflammatory cells that secrete cytokines and chemokines, leading to impaired healing process, thereby promoting fibrosis ultimately. ${ }^{22}$ A very recent study also mentioned the thickening of skin following the implantation of osmotic minipumps with BLM. ${ }^{19}$ We here further described the time course of changes in skin morphology, cutaneous infiltrates, and pro-inflammatory cytokine expression following the treatment of minipumps with BLM in detail. Thus, this modified murine model of SSc could be helpful to understand the underlying mechanisms of SSc and search for new therapeutic targets.

More importantly, the local cutaneous inflammatory response, elicited by BLM through osmotic minipumps, was characterized by the infiltration of leukocytes, including macrophages, neutrophils, and T cells (Figure 4). Considerable evidence has indicated that indeed type 2 polarized T-cell responses are important for fibrosis development and participate in SSc and related animal models. ${ }^{23}$ In present study, cytokine analysis showed that $\mathrm{CD} 4{ }^{+}$Th2 cells dominated in the T-cell infiltration: (1) the mRNA level of IL-4 was elevated at weeks 1 and 4 in the skin (Figure 5a); (2)
IL-13, in particular, acts as an effector molecule of Th2, and was shown to be increased at week 4 in the skin (Figure 5b); (3) Whereas significant pulmonary inflammation and fibrosis were induced, IL-4 started to be increased since the 4th week in the lung tissue following BLM treatment (Figure 6e). In addition, there was increased expression of the chemokine CCL17 (Figures 5b and 6d), the ligand of CCR4, which could recruit $\mathrm{CD} 4{ }^{+}$Th2 into the lesional sites. ${ }^{15,16}$ Therefore, these data collectively indicated that this modified model resembled the Th2-predominant pattern in SSc.

Interestingly, we found the accumulation of macrophages in the skin lesions after implantation of osmotic minipumps with BLM, whereas much fewer macrophages in the skin following implantation of minipumps with saline (Figure 4b). These data supported the involvement of macrophages in the initiation of SSc. In the skin from SSc patients, macrophages were observed between collagen bundles as well as around skin addenda and vessels, particularly in severely affected skin. ${ }^{24}$ Moreover, macrophages are classically activated towards the M1 and M2 phenotypes by their functions. ${ }^{25,26}$ M2 macrophages influence vascularization, wound repair, and have a critical role in fibrotic diseases by producing TGF- $\beta .{ }^{27,28}$ In the skin from localized SSc patients, CD163 ${ }^{+}$ or $\mathrm{CD} 204^{+} \mathrm{M} 2$ macrophages were reported to increase in the fibrotic areas, which is closely associated with the severity of inflammation. ${ }^{29}$ Therefore, in further study the phenotypes of macrophages in the local lesions need to be further distinguished. More importantly, the precise role of macrophages in SSc remains to be fully explored.

Another novel finding of this current study is that we found that a small number of $\mathrm{Gr}-1^{+}$neutrophils was present in the skin of SSc model, whereas the absence of Gr-1-positive cells was found in the skin of saline control (Figure 4c). In addition, the expression of CXCL2, the chemokine mainly responsible for recruiting neutrophils, ${ }^{13,14}$ was found to be increased in the skin lesion both at the early and late stages (Figures 5a and b). Accordingly, there was significantly increased numbers of neutrophils in the lesional skin of the patients with SSc compared with the normal skin of the health control. ${ }^{1}$ These data indicated the involvement of neutrophil in the progression of SSc. In addition, neutrophils could potentiate endothelial cell activation and apoptosis in endothelial cell-neutrophil co-cultures in presence of serum from SSc patients, and are largely mediated by IL- $6 .{ }^{30}$ Moreover, neutrophil was an important source of reactive oxygen species (ROS) that contributes to the inflammation in SSc. ${ }^{31-33}$ However, fine regulatory mechanism of neutrophil in SSc is far from clear. In this case, identifying functional significance of neutrophil in SSc may point to the mechanism of fibrogenesis and possible future target of pharmacological therapy for SSc.

Th17 cell, a subset of $\mathrm{T}$ helper cells, has an important role in tissue damage. It is recently proposed that IL-17 could be responsible for fibroblast proliferation and endothelium activation; ${ }^{2}$ therefore, it is hypothesized that a Th17 
deregulation could be a central component of SSc pathophysiology. However, in a more recent study, peripheral or local IL-17 amplification in SSc patients was not found. ${ }^{5}$ Instead, the authors found significantly increased IL-22 transcription levels in SSc lesional skin, as compared with healthy skin. In addition, flow cytometry confirmed the existence in SSc patients a distinct subset of $\mathrm{T}$ cells producing IL-22 in the absence of IL-17. ${ }^{5}$ In the present study, IL-22, but not IL-17, was shown to be significantly increased following BLM treatment (Figures $5 \mathrm{a}$ and $\mathrm{b}$ ). Therefore, the observation that IL-22, but not IL-17 transcript, was found to be upregulated in the skin samples of SSc model strongly suggested the presence of IL-22-producing cells and its possible association with SSc.

In summary, we have established a murine model by implanting an osmotic minipump containing BLM that led to tissue inflammation and fibrosis in the local skin and the distant lung, closely resembling human SSc with ILD-like manifestation. It is therefore anticipated that this model may provide advantages to investigate immunological mechanisms and test therapeutic interventions in SSc-ILD.

\section{ACKNOWLEDGMENTS}

We would like to thank Youfa Zhu for his help with the

immunohistochemistry techniques. This work is supported by the National Natural Science Foundation of China Grant 81373213 (to HZ), 81322043 (to RH) and Shanghai 'Shuguang Program' (to RH).

\section{DISCLOSURE/CONFLICT OF INTEREST}

The authors declare no conflict of interest.

1. Hussein MR, Hassan $\mathrm{HI}$, Hofny ER, et al. Alterations of mononuclear inflammatory cells, CD4/CD8 + T cells, interleukin 1beta, and tumour necrosis factor alpha in the bronchoalveolar lavage fluid, peripheral blood, and skin of patients with systemic sclerosis. J Clin Pathol 2005:58:178-184.

2. Deleuran B, Abraham DJ. Possible implication of the effector CD4+ T-cell subpopulation $\mathrm{TH} 17$ in the pathogenesis of systemic scleroderma. Nat Clin Pract Rheumatol 2007;3:682-683.

3. Kurasawa $\mathrm{K}$, Hirose $\mathrm{K}$, Sano $\mathrm{H}$, et al. Increased interleukin-17 production in patients with systemic sclerosis. Arthritis Rheum 2000;43:2455-2463.

4. Murata M, Fujimoto $M$, Matsushita $T$, et al. Clinical association of serum interleukin-17 levels in systemic sclerosis: is systemic sclerosis a Th17 disease? J Dermatol Sci 2008;50:240-242.

5. Mathian A, Parizot C, Dorgham K, et al. Activated and resting regulatory $T$ cell exhaustion concurs with high levels of interleukin-22 expression in systemic sclerosis lesions. Ann Rheum Dis 2012;71: 1227-1234.

6. Truchetet ME, Brembilla NC, Montanari E, et al. Interleukin-17A + cell counts are increased in systemic sclerosis skin and their number is inversely correlated with the extent of skin involvement. Arthritis Rheum 2013;65:1347-1356.

7. Gerber EE, Gallo EM, Fontana SC, et al. Integrin-modulating therapy prevents fibrosis and autoimmunity in mouse models of scleroderma. Nature 2013;503:126-130.

8. Hinchcliff M, Huang CC, Wood TA, et al. Molecular signatures in skin associated with clinical improvement during mycophenolate treatment in systemic sclerosis. J Invest Dermatol 2013;133: 1979-1989.
9. Yamamoto T, Takagawa S, Katayama I, et al. Animal model of sclerotic skin. I: Local injections of bleomycin induce sclerotic skin mimicking scleroderma. J Invest Dermatol 1999;112:456-462.

10. Akhmetshina A, Dees $C$, Busch $N$, et al. The cannabinoid receptor $C B 2$ exerts antifibrotic effects in experimental dermal fibrosis. Arthritis Rheum 2009;60:1129-1136.

11. Ashcroft T, Simpson JM, Timbrell V. Simple method of estimating severity of pulmonary fibrosis on a numerical scalE. J ClinPathol 1988:41:467-470.

12. Wynn TA. Fibrotic disease and the $T(H) 1 / T(H) 2$ paradigm. Nat Rev Immunol 2004;4:583-594.

13. Marcos V, Zhou Z, Yildirim AO, et al. CXCR2 mediates NADPH oxidaseindependent neutrophil extracellular trap formation in cystic fibrosis airway inflammation. Nat Med 2010;16:1018-1023.

14. De Filippo K, Dudeck A, Hasenberg M, et al. Mast cell and macrophage chemokines CXCL1/CXCL2 control the early stage of neutrophil recruitment during tissue inflammation. Blood 2013;121:4930-4937.

15. Kusumoto $M, X u B H$, Shi MY, et al. Expression of chemokine receptor CCR4 and its ligands (CCL17 and CCL22) in murine contact hypersensitivity. J Interferon Cytok Res 2007;27:901-910.

16. Mariani M, Lang R, Binda $E$, et al. Dominance of CCL22 over CCL17 in induction of chemokine receptor CCR4 desensitization and internalization on human Th2 cells. Eur J Immunol 2004;34:231-240.

17. Prescott RJ, Freemont $\mathrm{AJ}$, Jones $\mathrm{CJ}$, et al. Sequential dermal microvascular and perivascular changes in the development of scleroderma. J Pathol 1992;166:255-263.

18. Kalogerou A, Gelou E, Mountantonakis S, et al. Early T cell activation in the skin from patients with systemic sclerosis. Ann Rheum Dis 2005;64:1233-1235.

19. Lee $R$, Reese $C$, Bonner $M$, et al. Bleomycin delivery by osmotic minipump: similarity to human scleroderma interstitial lung disease. Am J Physiol Lung Cell Mol Physiol 2014;306:L736-L748.

20. Harrison Jr. JH, Lazo JS. High dose continuous infusion of bleomycin in mice: a new model for drug-induced pulmonary fibrosis. J Pharmacol Exp Ther 1987;243:1185-1194.

21. Lazo JS, Humphreys CJ, LACK OF. Metabolism as the biochemical basis of bleomycin-induced pulmonary toxicity. Proc Natl Acad Sci USA 1983;80:3064-3068.

22. King Jr. TE, Pardo A, Selman M. Idiopathic pulmonary fibrosis. Lancet 2011;378:1949-1961.

23. Belperio JA, Dy M, Burdick MD, et al. Interaction of IL-13 and C10 in the pathogenesis of bleomycin-induced pulmonary fibrosis. Am J Resp Cell Mol Biol 2002;27:419-427.

24. Ishikawa O, Ishikawa H. Macrophage infiltration in the skin of patients with systemic-sclerosis. J Rheum 1992;19:1202-1206.

25. Gordon S. Alternative activation of macrophages. Nat Rev Immunol 2003;3:23-35.

26. Mosser DM. The many faces of macrophage activation. J Leuk Biol 2003;73:209-212.

27. Raesl G, Beschinl A, Ghassabeh GH, et al. Alternatively activated macrophages in protozoan infections. Curr Opin Immunol 2007;19: 454-459.

28. Murray PJ, Wynn TA. Protective and pathogenic functions of macrophage subsets. Nat Rev Immunol 2011;11:723-737.

29. Higashi-Kuwata N, Makino $T$, Inoue $Y$, et al. Alternatively activated macrophages (M2 macrophages) in the skin of patient with localized scleroderma. Exp Dermatol 2009;18:727-729.

30. Barnes TC, Spiller DG, Anderson ME, et al. Endothelial activation and apoptosis mediated by neutrophil-dependent interleukin 6 transsignalling: a novel target for systemic sclerosis? Ann Rheum Dis 2011;70:366-372.

31. Czirjak L, Danko K, Sipka S, et al. Polymorphonuclear neutrophil function in systemic sclerosis. Ann Rheum Dis 1987;46:302-306.

32. Barnes TC, Anderson ME, Edwards SW, et al. Neutrophil-derived reactive oxygen species in SSc. Rheumatology (Oxford) 2012;51: 1166-1169.

33. Maugeri $N$, Rovere-Querini $P$, Baldini $M$, et al. Oxidative stress elicits platelet/leukocyte inflammatory interactions via HMGB1: a candidate for microvessel injury in sytemic sclerosis. Antioxid Redox Signal 2014:20:1060-1074. 